\title{
Quantitative metabolomics of the thermophilic methylotroph Bacillus methanolicus
}

\author{
Marc Carnicer ${ }^{1,2,3}$, Gilles Vieira ${ }^{1,2,3}$, Trygve Brautaset ${ }^{4}$, Jean-Charles Portais ${ }^{1,2,3}$ and Stephanie Heux $x^{1,2,3,5^{*}}$
}

\begin{abstract}
Background: The gram-positive bacterium Bacillus methanolicus MGA3 is a promising candidate for methanol-based biotechnologies. Accurate determination of intracellular metabolites is crucial for engineering this bacteria into an efficient microbial cell factory. Due to the diversity of chemical and cell properties, an experimental protocol validated on B. methanolicus is needed. Here a systematic evaluation of different techniques for establishing a reliable basis for metabolome investigations is presented.
\end{abstract}

Results: Metabolome analysis was focused on metabolites closely linked with B. methanolicus central methanol metabolism. As an alternative to cold solvent based procedures, a solvent-free quenching strategy using stainless steel beads cooled to $-20^{\circ} \mathrm{C}$ was assessed. The precision, the consistency of the measurements, and the extent of metabolite leakage from quenched cells were evaluated in procedures with and without cell separation. The most accurate and reliable performance was provided by the method without cell separation, as significant metabolite leakage occurred in the procedures based on fast filtration. As a biological test case, the best protocol was used to assess the metabolome of B. methanolicus grown in chemostat on methanol at two different growth rates and its validity was demonstrated.

Conclusion: The presented protocol is a first and helpful step towards developing reliable metabolomics data for thermophilic methylotroph B. methanolicus. This will definitely help for designing an efficient methylotrophic cell factory.

Keywords: Bacillus methanolicus, Methanol, Quantitative metabolomics, Quenching

\section{Background}

Industrial biotechnology mainly uses sugars and molasses as carbon sources. These raw materials come from plants and require cultivable land which is increasingly needed to produce food for human populations. The possibility of using non-food raw materials, such as onecarbon (C1) substrates, as alternative feedstock in microbial fermentation for the manufacturing of special, fine, bulk, and fuel chemicals has attracted considerable biotechnological and scientific interest. C1 compounds such as methane and methanol occur abundantly throughout

\footnotetext{
*Correspondence: stephanie.heux@insa-toulouse.fr

${ }^{5}$ LISBP-INSA de Toulouse, 135 Avenue de Rangueil,

31077 Toulouse Cedex 04, France

Full list of author information is available at the end of the article
}

nature, and in contrast to molasses, methanol is a pure raw material which can be completely consumed by methylotrophic bacteria during fermentation [1].

Methylotrophic bacteria have already been identified as potential producers for biotechnological processes [1]. Among the different microorganisms able to use $\mathrm{C} 1$ sources, the gram-positive facultative methylotroph Bacillus methanolicus is a possible cell factory for the industrial production of L-lysine, L-glutamate and cadaverine from methanol at elevated temperatures [2-4]. B. methanolicus MGA3 (ATCC 53907) growths at $50{ }^{\circ} \mathrm{C}$ and assimilates methanol by using the ribulose monophosphate (RuMP) pathway [5]. Genes involved in this metabolic pathway are located in the pBM19 plasmid and are upregulated upon growth on methanol 
compared to mannitol [6]. Recently, different isoenzymes present in the RuMP pathway have been biochemically characterized providing evidence for the importance of plasmidic isoenzymes in methanol-based growth [7-9]. The $B$. methanolicus genome sequence is now complete and provides physiological and metabolic traits that pave the way for system-level metabolic engineering [6]. Both proteome and transcriptome analyses have recently been used to analyse global gene regulation upon methylotrophic growth in this organism [10,11]. However, there are still gaps in our biochemical and regulatory understanding of how $B$. methanolicus efficiently uses methanol as sole source of carbon and energy.

Metabolomics is defined as the comprehensive analysis of the metabolites produced by an organism. Because the metabolome is the consequence of the amplification and integration of the other 'omic' levels, it provides information on cellular activity and instantaneous snapshots of cell physiology [12]. In addition, as metabolites are the functional entities within cells, genetic modifications or changes in the cell's environment have a direct influence on their levels. Therefore, assessing changes in metabolite levels in wild type vs. engineered strains or under different culture conditions may help elucidate limiting or inhibiting biosynthetic steps as well as advance our understanding of cellular metabolism [13]. This explain why metabolomics has become a major tool in metabolic engineering for strain improvement.

All procedures used for metabolome analysis have the same operational sequence, i.e. culture broth is sampled, cell metabolism is quenched and the metabolites are extracted from the cells. Because of the rapid biological turnover of the metabolites (from subseconds to $100 \mathrm{~s}$ ), the sampling and quenching steps have to be fast to properly stop the metabolic activity of the organism. Rapid sampling of the culture broth in a bioreactor can be achieved manually or automatically with sampling devices and stopped flow sampling systems [14-16]. Several quenching procedures exists which can be divided into two main groups: with or without cell separation. In the first group, the sample is mixed with cold solvent and the cells are separated from the culture medium by centrifugation or filtration $[17,18]$. The cells are then washed and resuspended in extraction solution (i.e. cold or hot solvent) $[14,15]$. The main limitation of such approaches is the leakage of intracellular metabolites into the solution due to damage to the membrane and cell walls when the cells are in contact with the quenching solution [19]. The extent of leakage is determined by different factors including time of exposure, quenching temperature, the properties of the cold aqueous methanol solution (e.g. ionic strength, concentration of methanol; [17]) and the physical-chemical properties of the metabolites (e.g. size and polarity; [20]). To prevent leakage, fast filtration methods collect cells before quenching [16]. In this case, the cells are first separated from the culture medium by vacuum filtration, the filter is then washed using an appropriate solution to get rid of extracellular medium, and the cells are then transferred into cold organic solvent to quench the metabolism [16, 21]. However, these methods do not allow immediate quenching of metabolic activity [19, 21, 22]. In the second group, the cell separation step is skipped, and quenching and extraction are performed simultaneously using appropriate buffers $[15,21]$. This can even be extended to a fully integrated approach which allows quenching and extraction during sampling [23]. In contrast to cell separation methods, which give direct access to the levels of intracellular metabolites, here, the levels of extracellular metabolites have to be quantified $[15,21]$. In these approaches, the levels of metabolites in the cells are estimated by the socalled "differential method" by subtracting the amount in the extracellular medium from the total amount in the whole broth. Using such integrated procedures circumvents leakage phenomena while allowing a sub-second arrest of metabolic activity; however, the precision of the measurements of intracellular metabolites is significantly reduced $[19,21]$.

Unfortunately, due to the vast diversity of chemical and cell properties, no universal method exists for metabolome analysis of bacterial cells, and protocols have to be adapted and evaluated for each individual organism [17, $18,21,24]$. In this study, we established the first method for proper metabolome quantification in B. methanolicus. A solvent-free quenching strategy using stainless steel beads cooled to $-20{ }^{\circ} \mathrm{C}$ was assessed as a possible alternative to cold solvent based approaches [25]. The cold stainless steel bead sampling method has been proven to be suitable when rapid sampling and arrest of cells are required when following the dynamics of substrate uptake rate in the short sampling time frame [25, 26]. Cold beads can thus be a good strategy for efficient metabolic arrest for quantifying intracellular metabolites pools. The applicability of protocols with and without cell separation was evaluated with respect to their precision, the consistency of the measurements, and the extent of leakage of metabolites from quenched cells. To this end, a quantitative mass balance approach was used to monitor the fate of metabolites during processing of the samples $[17,18]$. The best protocol was then used to differentiate the relevant metabolome of B. methanolicus MGA growing on methanol at two different growth rates, thereby providing new valuable insights into the methylotrophic properties of this bacterium. 


\section{Methods}

\section{Strain and culture conditions}

All analytical grade reagents were supplied by Sigma-Aldrich In this study, the gram-positive methylotrophic bacterium B. methanolicus wild-type MGA3 (ATCC 53907) strain was used. Chemostat cultures were performed in 0.5 litre bioreactors (INFORS HT Multifors, The Netherlands) with a working volume of 0.4 litres, coupled to a Dycor ProLine Process Mass Spectrometer (AMETEK Process Instruments, USA). The culture medium was derived from [27]. The medium per litre in shake flask pre-cultures and in the batch phase of the cultures was: $8.42 \mathrm{~g} \mathrm{Na}_{2} \mathrm{HPO}_{4} \cdot 12 \mathrm{H}_{2} 0,1.47 \mathrm{~g} \mathrm{KH}_{2} \mathrm{PO}_{4}, 2.11 \mathrm{~g}$ $\left(\mathrm{NH}_{4}\right)_{2} \mathrm{SO}_{4}, 0.25$ g yeast extract, $1 \mathrm{ml}$ of $1 \mathrm{M} \mathrm{MgSO}$ solution, $1 \mathrm{ml}$ of trace salt solution, $1 \mathrm{ml}$ of vitamin solution, $0.05 \mathrm{ml}$ Antifoam 204 (Sigma-Aldrich) and $120 \mathrm{mM}$ of methanol. The trace salt solution per litre was: $5.56 \mathrm{~g} \mathrm{FeSO}_{4} \cdot 7 \mathrm{H}_{2} \mathrm{O}, 0.027 \mathrm{~g} \mathrm{CuCl}_{2} \cdot 2 \mathrm{H}_{2} \mathrm{O}, 7.35$ $\mathrm{CaCl}_{2} \cdot 2 \mathrm{H}_{2} \mathrm{O}, 0.040 \mathrm{~g} \mathrm{CoCl} \cdot 6 \mathrm{H}_{2} \mathrm{O}, 9.90 \mathrm{~g} \mathrm{MnCl}_{2} \cdot 4$ $\mathrm{H}_{2} \mathrm{O}, 0.288 \mathrm{~g} \mathrm{ZnSO}_{4} \cdot 7 \mathrm{H}_{2} \mathrm{O}$ and $0.031 \mathrm{~g} \mathrm{H}_{3} \mathrm{BO}_{3}$. The vitamin solution per litre was: $0.10 \mathrm{~g}$ D-biotin, $0.10 \mathrm{~g}$ thiamine $\cdot \mathrm{HCl}, 0.10 \mathrm{~g}$ riboflavin, $0.10 \mathrm{~g}$ pyridoxine $\cdot \mathrm{HCl}$, $0.10 \mathrm{~g}$ pantothenate, $0.10 \mathrm{~g}$ nicotinamide, $0.02 \mathrm{~g}$ p-aminobenzoic acid, $0.01 \mathrm{~g}$ folic acid, $0.01 \mathrm{~g}$ vitamin B12 and $0.01 \mathrm{~g}$ lipoic acid. The culture medium used in the continuous phase was the same as in the batch phase but without the yeast extract.

First, half litre shake flasks containing $150 \mathrm{ml}$ of the pre-culture medium were inoculated with cryostock of $B$. methanolicus cells. The cultures were grown overnight at $50{ }^{\circ} \mathrm{C}$ under shaking at $200 \mathrm{rpm}$, and used to inoculate the reactors. After complete termination of the batch phase, approximately $7 \mathrm{~h}$ after inoculation, the chemostat phase was started. In this phase, the cells were grown under carbon-limited conditions at specific dilution rates (D), $0.10 \mathrm{~h}^{-1}$ or $0.15 \mathrm{~h}^{-1}$. The aeration rate of $1 \mathrm{vvm}$ was controlled by a mass flow meter (INFORS HT Multifors, The Netherlands) and $\mathrm{pO}_{2}$ was maintained above $25 \%$ throughout culture. Temperature, $\mathrm{pH}$ and stirring speed were maintained at $50{ }^{\circ} \mathrm{C}, \mathrm{pH} 6.5$ (with $\mathrm{KOH} 1 \mathrm{M}$ ) and $800 \mathrm{rpm}$, respectively. The $\mathrm{N}_{2}, \mathrm{O}_{2}$, Argon, $\mathrm{CO}_{2}$ and methanol concentrations in the bioreactor off-gas were measured on-line with the mass spectrometer.

\section{Sampling, quenching and extraction}

Figure 1 shows the samples taken to evaluate the methods for metabolome quantification in B. methanolicus. Whatever the type of sample, quenching was done using seven pre-cooled $\left(-20{ }^{\circ} \mathrm{C}\right.$ if not stated differently) stainless steel beads ( $4 \mathrm{~mm}$ diameter, Saluc, Belgium). The mass of beads used per mass of liquid ranged between 4 and $10 \mathrm{~g} / \mathrm{g}$ depending on the volume sampled from the culture. All the metabolites were extracted using $3 \mathrm{ml}$ of a solution containing acetonitrile : methanol : $0.1 \mathrm{M}$ formic acid, 40:40:20 v/v and left at $-20{ }^{\circ} \mathrm{C}$ for $1 \mathrm{~h}$. All the extracted samples were stored at $-80{ }^{\circ} \mathrm{C}$ until further treatment.

Quenched whole broth (WB) samples Samples were taken directly from the bioreactor using a $1 \mathrm{ml}$ syringe containing seven pre-cooled $\left(-20{ }^{\circ} \mathrm{C}\right)$ stainless steel beads. The samples were then placed in a tube containing the extracting solution and vortexed to homogenise the sample. The exact volumes sampled were determined by weighing the extracting tubes before and after sampling. U- ${ }^{13}$ C-labeled Escherichia coli cell extract $(200 \mu \mathrm{l})$ was added to the extracting tube as internal standard. Three technical replicates per culture were performed. The average time between sample collection and extraction was $21 \pm 2 \mathrm{~s}$.

Quenched culture filtrate (CF) samples Culture filtrate samples were obtained using a $10 \mathrm{ml}$ syringe attached to a $0.20 \mu \mathrm{m}$ filter (Minisart, Sartorius). The samples were directly filtered during their removal from the bioreactor sampling port. Seven pre-cooled $\left(-20{ }^{\circ} \mathrm{C}\right)$ stainless steel beads were placed inside the syringe. Next, the filtrate solution was moved to an ice-cooled Eppendorf tube from which $200 \mu \mathrm{l}$ was dispensed into an extracting tube together with $200 \mu \mathrm{l}$ of $\mathrm{U}_{-}{ }^{13} \mathrm{C}$-labeled $E$. coli cell extract. Three technical replicates per culture were performed. The average time between sample collection and extraction was $46 \pm 1 \mathrm{~s}$.

Quenched and washed cells separated from the medium by filtration (QC) and washing solution (WS) samples These samples were taken in the same way as the WB samples and transferred to ice-cooled Eppendorf tubes using syringes containing seven stainless steel beads precooled to $-20{ }^{\circ} \mathrm{C}$ (QC-20) or to $4{ }^{\circ} \mathrm{C}$ (QC-4). From the Eppendorf tubes, $200 \mu \mathrm{l}$ of culture were transferred to membrane disk filters $(0.2 \mu \mathrm{m}$ pore size, Sartolon Polyamid, Sartorius) and filtered using a vacuum pump. The filters were washed twice with $1 \mathrm{ml}$ of cooled $\left(4{ }^{\circ} \mathrm{C}\right)$ medium without vitamins, trace salts or methanol. After washing, the filters were rapidly transferred to extracting tubes together with $200 \mu \mathrm{l}$ of $\mathrm{U}^{13}{ }^{13} \mathrm{C}$-labeled $E$. coli cell extract. The washing solution for the two quenching temperatures $\left(4^{\circ} \mathrm{C}\right.$ and $\left.-20^{\circ} \mathrm{C}\right)$ was collected and mixed with $200 \mu \mathrm{l}$ of U- ${ }^{13} \mathrm{C}$-labeled $E$. coli cell extract (WS-4 and WS-20). Duplicate technical samples were taken in both protocols tested (QC-20 + WS-20 and QC-4 + WS-4). The average time between sample collection and extraction was $105 \pm 2 \mathrm{~s}$. 


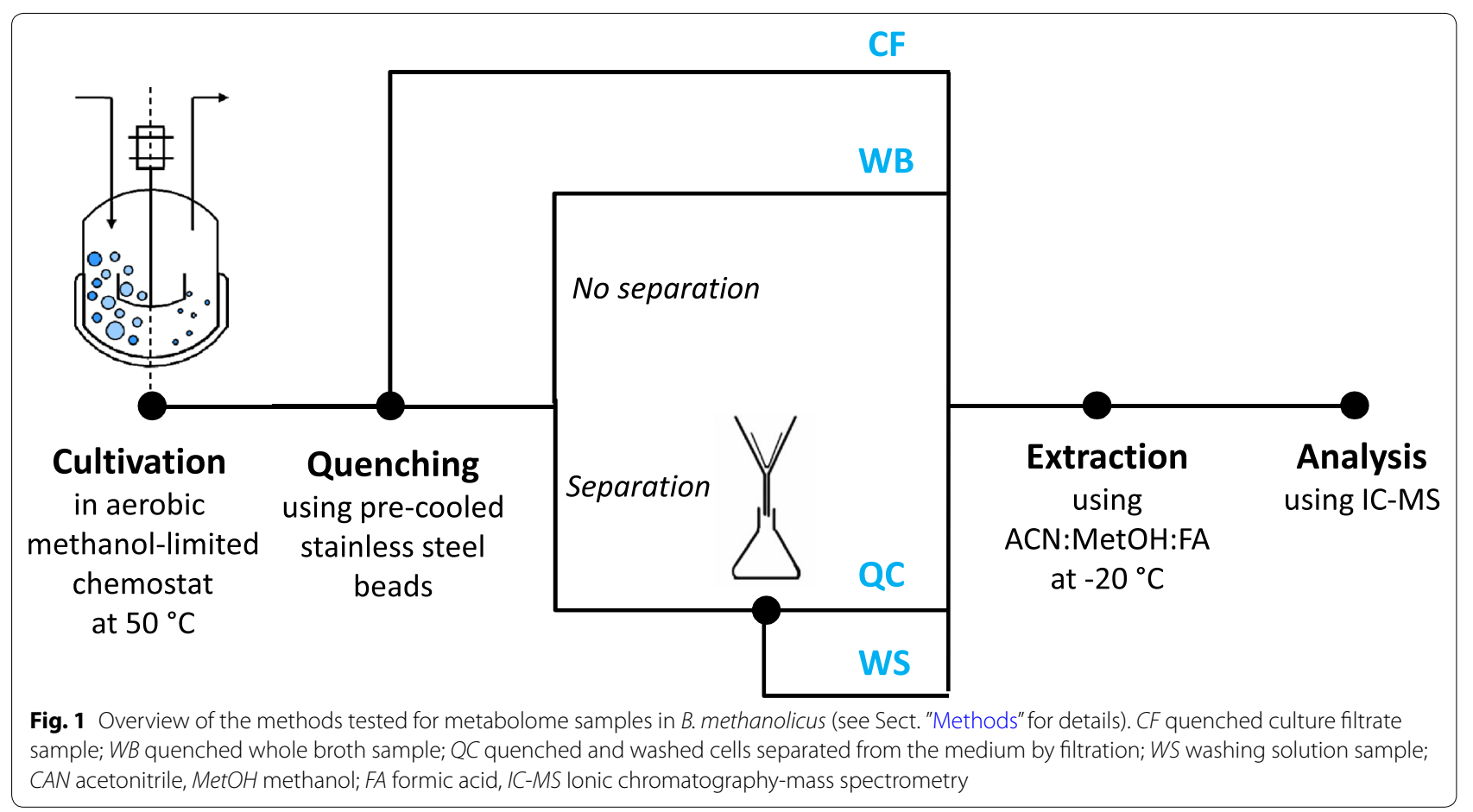

\section{Measurements of intracellular metabolite levels}

Extracted samples were evaporated in a Rotavapor (Büchi, Switzerland) for approximately $15 \mathrm{~h}$ until complete dryness. The samples were then re-dissolved in $400 \mu \mathrm{l}$ of ultrapure water and stored at $-20{ }^{\circ} \mathrm{C}$ until analysis. Further dilutions were performed, if needed, to adjust the salt content of the samples to avoid ion suppression during MS analysis.

Metabolome analysis Intracellular metabolites content of the WB, CF, QC and WS samples were analyzed as previously described [21, 28]. Briefly a Dionex ICS 2000 system (Dionex, Sunnyvale, USA) coupled to a triple quadrupole QTrap 4000 mass spectrometer (Applied Biosystems, Foster City, USA) was used. Peak integration was performed manually using Analyst 1.5.2 software (Sierra Analytics, USA). All samples were analyzed in the negative mode by multiple reactions monitoring (MRM). To ensure highly accurate quantification, the isotope dilution mass spectrometry (IDMS) method was used [29]. Integration of all the peaks were performed manually using the Analyst 1.5.2 software (Sierra Analytics, USA). The number of samples collected and analysed for each experiment is given in Additional file 1.

Metabolome quantification The quantification of intracellular metabolites in all biological samples was done using a program developed in $\mathrm{R}$ [30]. Briefly, a calibration curves $\left({ }^{12} \mathrm{C}\right.$ analyte peak area $/{ }^{13} \mathrm{C}$ analyte peak area) were performed using 8 concentrations ranging from $0.04 \mu \mathrm{M}$ until $10.0 \mu \mathrm{M}$. The concentration (Ce) of each analyte in $(\mu \mathrm{mol} / \mathrm{L})$ in the extract was determined using the following equation: $\mathrm{Ce}=(\mathrm{y}-\mathrm{b}) / \mathrm{a}$ with $\mathrm{y}={ }^{12} \mathrm{C}$ peak area $/{ }^{13} \mathrm{C}$ peak area. The calibration coefficients [i.e. the slope (a), the intercept (b) and the correlation coefficient $\left(r^{2}\right)$ ] for each analyte are given in Additional file 1 . The metabolite concentrations in $\mu \mathrm{mol} / \mathrm{gDCW}$ for each replicate were calculated using the dry cell weight measurement and the weighted sample volume taken from the bioreactor as described in Additional file 1. The mean of concentrations of each metabolite and the standard error were calculated for each sample type. For the differential method, the intracellular concentration was obtained by the difference between the WB and CF and the error was obtained by propagation on the standard error.

Our analysis of the metabolome of $B$. methanolicus focused on metabolites closely related with the central methanol metabolism. The complete list is given in Additional file 1 .

\section{Measurements of extracellular metabolite levels}

Initial culture media and culture samples collected at different times were filtered (Minisart 0.2 $\mu \mathrm{M}$ filter from Sartorius, Göttingen, Germany). Supernatant fraction were prepared for 1D 1H NMR analysis, by adding 100 $\mu \mathrm{L}$ of Deuterated trimethylsilyl propionate (TSPd4) at 4,3 mM diluted in $\mathrm{DH}_{2} \mathrm{O}$ to $500 \mu \mathrm{L}$ of supernatant. 1D $1 \mathrm{H}$ NMR spectra were acquired on a Bruker Ascend 
$800 \mathrm{MHz}$ magnet (Bruker, Germany) using a $5 \mathrm{~mm}$ CPQCI cryoprobe $1 \mathrm{H}-31 / 13 \mathrm{C} / 15 \mathrm{~N} / \mathrm{Z}$ GRD. A sequence using presaturation (ZGPR) was used for water signal suppression, with a $30^{\circ}$ pulse angle and a relaxation delay between scans of $10 \mathrm{~s}$ to ensure full signal recovery. A total of 32 scans were accumulated (after 4 dummy scans) with $292 \mathrm{~K}$ data points, $6.83 \mathrm{~s}$ of acquisition time, $5 \mathrm{~s}$ of recycle delay and no spin. Using Topspin 2.1 (Bruker, Rheinstatten, Germany), the FIDs were zero-filled, Fourier transformed with $0.5-\mathrm{Hz}$ exponential line broadening, manually phase corrected, automatically baseline corrected, and aligned to the TSPd4 signal. Topspin 2.1 (Bruker, Rheinstatten, Germany) was also used for peak integration. Metabolite quantification was performed using a program developed in R [30]. Three samples were collected and analyzed for each dilution rate.

\section{Dry cell weight (DCW) analysis}

Samples $(3 \times 5 \mathrm{ml})$ of culture broth were taken from the bioreactors and filtered with a pre-weighted membrane disk filter $(0.2 \mu \mathrm{m}$ pore size, Sartolon Polyamid, Sartorius) using a vacuum pump. No washing steps were performed to remove salts from the biomass because the chemostat medium contains $11.9 \mathrm{~g} / \mathrm{l}$ of salt (see above) which is close to isotonic $(0.9 \%)$. The membrane disk filters were dried at $80^{\circ} \mathrm{C}$ until stable weight.

\section{Data consistency}

Chemostat cultures Under the culture conditions applied in this study, B. methanolicus biomass and carbon dioxide were the only compounds produced. The consistency of the experimental data was checked using standard data reconciliation procedures under the elemental mass balance constraint [31, 32]. For all chemostat cultures, consistency was acceptable at a confidence level of $95 \%$, showing there were no gross measurement errors. The biomass elemental composition used in the reconciliation procedure was taken from the closely related non-methylotrophic bacterium Bacillus subtilis, $\mathrm{CH}_{1.646} \mathrm{~N}_{0.219} \mathrm{O}_{0.410} \mathrm{~S}_{0.005}$ [33]. Ash contents were considered to be $6 \%$ of the dry cell weight, which is the average value obtained from different microorganisms (i.e. Escherichia coli, Aspergillus niger, Penicicillium chrysogenum, Klebsiella aerogenes) [34].

Consistency of metabolite measurements The consistency of the metabolome data obtained was checked under the constraint of Eq. 1, as described in [18]. Briefly, the amount of each metabolite was quantified in the different samples (WB, QC, CF and WS). Accordingly, mass balance should be satisfied for each metabolite, $i$, independently of the sampling method used:

$$
\mathrm{M}_{\mathrm{i}}(\mathrm{WB})=\mathrm{M}_{\mathrm{i}}(\mathrm{QC})+\mathrm{M}_{\mathrm{i}}(\mathrm{WS})
$$

This balance states that the total amount of each metabolite (i.e. the extracellular + intracellular amount) present in WB samples should be equal to the sum of $\mathrm{QC}$ and WS measurements. The redundancy of the data set makes it possible to check the statistical consistency using the calculated $x^{2}$-distributed consistency index $h$ [31]. In our conditions, the threshold value of the $h$ index with $95 \%$ of confidence level is 9.49. An $h$ index above this value is proof of a gross measurement error. All the calculations were performed using $\mathrm{R}$ [30].

\section{Results and discussion}

\section{Cell quenching by solvent free method}

Traditionally, cellular metabolism is quenched using cold methanol solvent $[17,21]$. However, quenching a methylotrophic metabolism using methanol can affect the results due to its potential assimilation. In addition, this method is clearly not appropriate for gram positive bacteria because it causes intracellular metabolites to leak from the cells into the quenching solution [21]. As an alternative, we evaluated the capacity of pre-cooled steel beads [25] to quench $B$. methanolicus metabolism. In this method, metabolism is supposed to be quenched by exposing the culture broth to stainless steel beads (4-mm diameter) in a syringe precooled to $-20^{\circ} \mathrm{C}$. In our conditions, this should induce a sudden drop in temperature of approximately $40-50{ }^{\circ} \mathrm{C}$, which has been reported to halt metabolism in other organisms $[15,17,18]$.

To evaluate the feasibility of this quenching procedure, whole broth samples (WB) from a B. methanolicus chemostat culture growing at D: $0.10 \mathrm{~h}^{-1}$ were taken in triplicate using a $1 \mathrm{ml}$ syringe containing pre-cooled $\left(-20^{\circ} \mathrm{C}\right)$ stainless steel beads (Fig. 1). Considering that metabolite turnover times ranged from a sub-second to seconds and that $21 \mathrm{~s}$ were required to process the samples (i.e. from sampling to the extraction step), if enzymatic reactions were still taking place (even at very low rates), the reproducibility of WB samples would be affected. The size of the metabolite pool and the standard error (SE) measured in WB samples are listed in Table 1. As expected, differences between the intracellular metabolite pools were observed as a result of the $B$. methanolicus metabolism. As previously reported for Corynebacterium glutamicum, Saccharomyces cerevisiae and Pichia pastoris, reproducibility was metabolite dependent [18]. 23PG and AMP showed the highest relative standard error (RSE) of $19.4 \%$ and $14.6 \%$, respectively. However, for the 18 metabolites analysed, the reproducibility of the measurements was on average less than $7 \%$, indicating no significant variation in the size of the metabolite pool. This demonstrates that metabolic activity is efficiently blocked by the cold stainless beads. This method was therefore used to quench B. methanolicus cells. 
Table 1 Metabolome content of WB samples collected from B. methanolicus MGA3 grown in methanol-limited chemostat at D: $0.10 \mathrm{~h}^{-1}$

\begin{tabular}{lcr}
\hline & $\boldsymbol{\mu m o l} / \mathbf{g D C W} \pm \mathbf{S E}$ & RSE \\
\hline 23PG & $6.1 \pm 1.2$ & 19.4 \\
6PG & $7.5 \pm 1.1$ & 14.3 \\
ADP & $7.23 \pm 0.10$ & 1.4 \\
AMP & $11.9 \pm 1.7$ & 14.6 \\
ATP & $9.6 \pm 0.4$ & 4.2 \\
Cit & $9.1 \pm 0.9$ & 10.4 \\
F1P & $0.9 \pm 0.1$ & 8.5 \\
F6P & $20.1 \pm 0.2$ & 1.1 \\
FBP & $15.9 \pm 1.2$ & 7.8 \\
Fum & $13.4 \pm 0.1$ & 0.8 \\
G6P & $23.0 \pm 3.1$ & 13.5 \\
M6P & $1.00 \pm 0.02$ & 2.3 \\
Mal & $8.77 \pm 0.13$ & 1.5 \\
PEP & $2.61 \pm 0.11$ & 4.2 \\
P-Ser & $0.31 \pm 0.02$ & 7.4 \\
R5P & $13.6 \pm 0.4$ & 2.8 \\
S7P & $12.3 \pm 0.7$ & 5.4 \\
Shi3P & $0.17 \pm 0.001$ & 0.8 \\
Mean & & 6.7 \\
\hline Sestanaterr & &
\end{tabular}

se standard error; rse relative standard deviation in \%

The metabolite abbreviations and the raw data are given in Additional file 1

\section{Separation versus no separation of cells prior extraction}

Once quenched with cold stainless beads, B. methanolicus cells can be separated from the cultivation medium prior to extraction or directly mixed with the extraction solution along with their culture medium. In terms of absolute quantification, both protocols have advantages and limitations and choosing one or the other will depend on the extent of leakage from the cells. Protocols based on cell separation make it possible to directly estimate the precise intracellular levels of all metabolites, even those that are largely excreted by the cells. However if leakages do occur, the levels of intracellular metabolites will be underestimated because some of the metabolites are lost in the discarded solutions (i.e. the quenching and washing solutions) $[17,21]$. Protocols without cell separation can be used even when leakage occurs, since the intracellular and extracellular metabolites are analysed together. In this case, the level of intracellular metabolites is determined indirectly by subtracting the concentration of extracellular metabolites measured in the culture filtrate from the total pool (intra + extra) in the whole broth sample. However this method can only be used with metabolites that occur in reasonable amounts outside the cell $[19,21]$.

To assess which protocols can be used to elucidate the metabolome of $B$. methanolicus, two independent chemsotat cultures were performed at D: $0.10 \mathrm{~h}^{-1}$ and different types of samples i.e. quenched whole broth (WB), culture filtrate (CF), quenched and washed cells separated from the medium by filtration (QC) and quenching and washing solution (WS) were collected when the culture was in steady state (see Fig. 1). Filtration was preferred to separate quenched cells from the cultivation medium because it has been reported to be more efficient in eliminating extracellular metabolites than centrifugation [35]. In addition, filtration is much faster than centrifugation, hence limiting the period during which metabolites can leak from cells by diffusion over the cell membrane [20]. To assess the sensitivity of $B$. methanolicus to cold shock, i.e. the sudden release of metabolites from the cells when the broth is rapidly cooled $[16,36]$, two quenching temperatures (i.e. $-20{ }^{\circ} \mathrm{C}$ and $4{ }^{\circ} \mathrm{C}$ ) were tested.

\section{Detection of metabolite leakage out of the cells}

To check for the occurrence of leakage, quantitative evaluation of the protocols was carried out by mass balance analysis, based on measurements of the metabolites in all the fractions. Figure 2 shows the mass balances for the two protocols and eight selected metabolites with different physical-chemical properties as an illustrative example (see Additional file 1 for complete dataset). Analysis of the different fractions revealed the presence of metabolites both in CF and WS fractions. The presence of metabolites in CF fraction is not surprising and has already been observed for several species [15, 22, 37]. This phenomenon called "extended" overflow metabolism is explained by a passive or active transportation of metabolites from the cells into in the cultivation medium due to a misbalance between carbon uptake and consumption during exponential growth [37]. However, the amount of metabolites in the WS fraction was much higher than in the CF fraction, indicating an additional loss of intracellular metabolites from the cells. This can be explained by the destruction of cell integrity during sample processing. In our experimental setting, cells damages can happen during the quenching step on the freezing stainless steel beads and/or during cell separation by filtration and washing (Fig. 1). Comparison of the extracellular fraction of metabolites obtained with (i.e. WCS $-20^{\circ}$ and WCS $+4^{\circ}$ ) or without (WOCS) cells separation indicated a higher fraction of extracellular metabolites for filtrated cells (Table 2). This was amplified when the quenching temperature was set to $4{ }^{\circ} \mathrm{C}$ (WCS $\left.+4^{\circ}\right)$. Together this data demonstrated that more intracellular metabolites leaves the cells during the filtration/washing procedures. Extend of leakage depended on the nature of the metabolites but was relatively high for the protocol with cell separation. On average $74 \%$ of the intracellular 


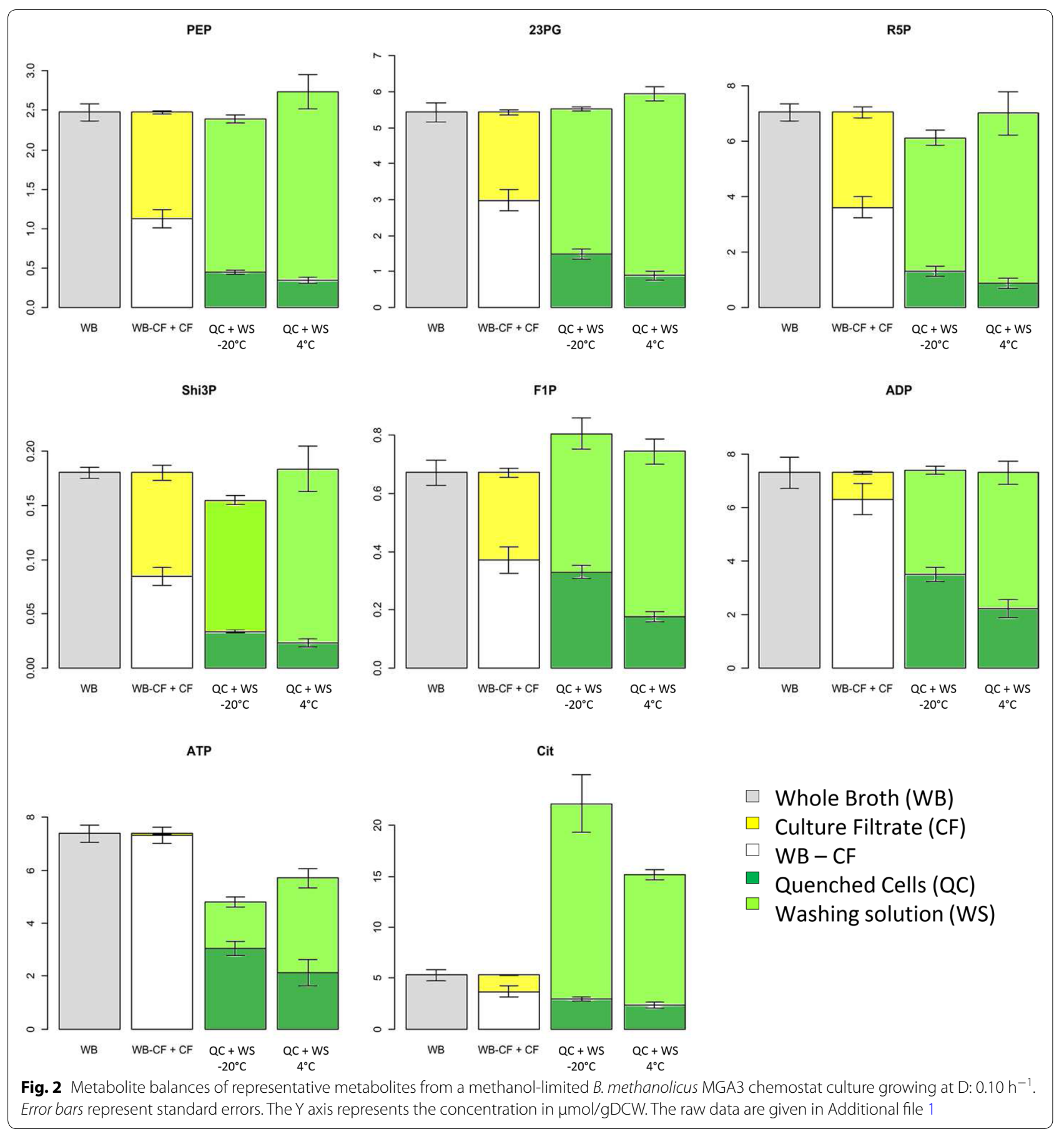

pool of metabolites were outside the cells against $50 \%$ for the protocol w/o cells separation. This resulted in an underestimation of the intracellular metabolites pools for the filtration based method (QC fraction) compared with the method without cells separation (WB-CF fraction) (Fig. 2).

We cannot exclude that metabolite leakage occurs also during the quenching procedure as stainless steel beads may cause double stress towards B. methanolicus, physical (crash within steel beads) and temperature, which both may affect the cell structure. This should be confirmed by analyzing the morphological status of the cells or by comparing the metabolite content of a non-quenched vs a quenched CF fraction. However, the fractions of extracellular pools varied across the different metabolites and some of them were really low, indicating that the presence 
Table 2 Calculated $x^{2}$-distributed consistency index $h, p$ value, extracellular fraction of the different metabolites analysed for the protocols with cells separation with a quenching temperature of $-20^{\circ}$ (i.e. WCS $-20^{\circ}$ ); with cells separation with a quenching temperature of $4{ }^{\circ} \mathrm{C}$ (i.e. WCS $+4^{\circ}$ ) and without cells separation with quenching temperature of $-20^{\circ}$ (WOCS $-20^{\circ} \mathrm{C}$ ) of the different metabolites analysed

\begin{tabular}{llllll}
\hline & \multicolumn{2}{l}{ Extracellular fraction in \% } & h index & $\boldsymbol{p}$ value \\
\cline { 2 - 4 } & WCS - 20 & WCS + $\mathbf{4}^{\text {oa }}$ & WOCS $-\mathbf{2 0}^{\text {ob }}$ & & \\
\hline 23PG & 71.5 & 80.4 & 47.5 & 1.71 & 0789 \\
6PG & 85.5 & 87.6 & 23.0 & 1.75 & 0781 \\
ADP & 53.9 & 64.8 & 14.0 & 0.03 & 1000 \\
AMP & 81.9 & 86.3 & 91.0 & 1.52 & 0823 \\
ATP & 31.7 & 54.5 & 1.0 & 13.10 & 0011 \\
Cit & 82.3 & 83.5 & 24.0 & 81.67 & $<0.0001$ \\
F1P & 63.4 & 74.0 & 52.5 & 5.13 & 0275 \\
F6P & 80.2 & 87.2 & 75.0 & 0.86 & 0930 \\
FBP & 38.2 & 61.7 & 10.0 & 1.21 & 0876 \\
Fum & 98.7 & 95.5 & 99.5 & 1.27 & 0866 \\
G6P & 91.6 & 93.5 & 84.5 & 0.03 & 1000 \\
M6P & 70.2 & 79.1 & 57.0 & 1.22 & 0875 \\
Mal & 92.1 & 93.1 & 89.5 & 4.96 & 0292 \\
PEP & 81.3 & 84.4 & 59.5 & 0.75 & 0945 \\
P-Ser & 70.0 & 81.4 & 17.0 & 0.10 & 0999 \\
R5P & 82.9 & 87.2 & 55.5 & 0.13 & 0998 \\
S7P & 76.9 & 87.6 & 47.5 & 1.65 & 0801 \\
Shi3P & 76.8 & 83.7 & 53.5 & 0.06 & 1000 \\
\hline
\end{tabular}

The metabolite abbreviations and the raw data are given in Additional file 1

a Ratio between concentrations in the washing solution and in the washing solution plus quenched and washed cells separated from the medium by filtration (WS *100)/(WS + QC)

b Ratio between concentrations in the culture filtrate and in the whole broth $\left(\mathrm{CF}^{*} 100\right) / \mathrm{WB}$

of these metabolic intermediates in the culture medium cannot be explained only by cell lysis. Finally the fractions of extracellular metabolites obtained with our quenching method were in the same range of the ones obtained for E. coli without stainless steel beads [22], suggesting that leakage may not only be caused by the beads. According to our data, fewer metabolites were released at lower quenching temperature, highlighting that in $B$. methanolicus, leakage may not be primarily caused by cold shock. Further investigation is thus required to identify the factor(s) responsible for metabolite leakage in $B$. methanolicus. Overall this results pinpointed significant metabolites leakage with cell separation method which is consistent with previous data [21]. However, the average time between sample collection and extraction for the method with cell separation is about double that for the method without cell separation. We thus cannot exclude that this extra timing can cause extra cell stress partially explaining the high metabolite leakage observed.

\section{Consistency of the measurements}

The consistency of each metabolite measurement was evaluated under the elemental mass balance constraint (Eq. 1 in Sect. "Methods") which should be fulfilled if the quantification is correct. Except for ATP and citrate (Cit), the $\mathrm{h}$ index was below 9.49 (i.e. the limit value to accept no mismatches on quantification), indicating that quantification was consistent whatever the protocol used (Table 2). Except for citrate and ATP, metabolite levels were almost similar in the WB fraction than in the QC + WS fractions indicating proper sampling and quantification. In the case of citrate, the gross error measurement can be explained by external contamination which led to higher amount of citrate in the WS fraction (Fig. 2), most probably from the filter. In contrast, the amounts of ATP measured in the cell extract plus the washing solution $(\mathrm{QC}+\mathrm{WS})$ were lower than the amounts measured in the WB, indicating loss of ATP during sample treatment, which could not be corrected by the use of fully labelled ATP as internal standard (Fig. 2). In addition, ATP was apparently not converted into ADP or AMP, since no significant increase in those pools was observed. Overall, the consistency test showed that 16 out of 18 metabolites tested for displayed no quantification mismatches, highlighting the quality of the measurements.

\section{Precision of the protocol without cell separation}

In previous sections, we demonstrated that when $B$. methanolicus cells are separated from the medium prior extraction, most of the metabolites are lost in the washing solution. Therefore the method without cell separation prior extraction is preferable for the measurement of its metabolome. However, the precision of the quantification using such approach is strongly affected by the concentration of metabolites outside the cells. The effect on measurement precision can be seen in Fig. 3. While separate measurements of $\mathrm{WB}$ and $\mathrm{CF}$ showed a relative standard error (RSE) below $20 \%$ (Fig. 3a), the RSE of intracellular metabolite levels obtained by combining the two measurements was higher (Fig. 3b). This was particularly true for metabolites found in significant amounts in the culture filtrates (i.e. outside the cell) such as glucose-6-phosphate (G6P), AMP and TCA intermediates (Table 2). However, for metabolites with extracellular fractions below 50-60 \%, RSE remained below $20 \%$.

\section{Test case: methanol derived metabolome analysis} under different growth rates

In this study we optimized a procedure for metabolomics analysis of the thermophilic methylotroph B. methanolicus. 

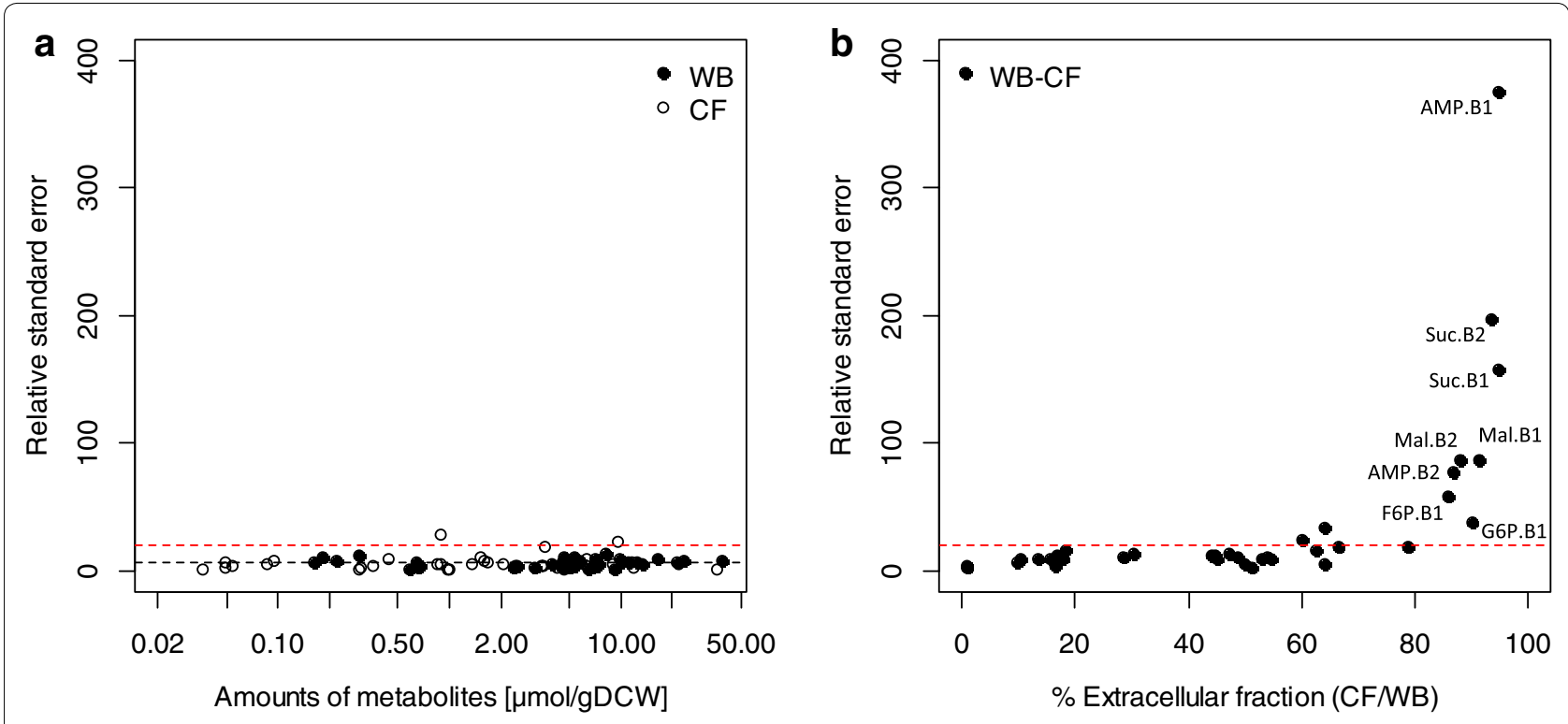

Fig. 3 Precision of the metabolite quantifications by differential method in B. methanolicus. a Precision of metabolite measurements performed on WB and CF samples. Black dashed line represents the average of WB and CF relative standard error. b Precision of intracellular pool measurements obtained using the differential method (WB-CF) versus the extracellular fraction of each specific metabolite. Red dashed line represents the relative standard deviation threshold for acceptable precision (i.e. $20 \%$ ). Fumarate values were not plotted because its RSE were over 400 . B1 and B2 state for biological replica 1 and 2. The raw data are given in Additional file 1

This procedure is based on the quenching the whole broth with cold stainless-steel beads and subsequent extraction of the metabolites in a cold methanol/acetonitrile solution. To validate our protocol, we applied it on four B. methanolicus cultivations on methanol in chemostat at two different growth rates, 0.10 and $0.15 \mathrm{~h}^{-1}$.

\section{Physiological parameters}

The rates of methanol and oxygen consumption and the rates of biomass and carbon dioxide production were calculated along with methanol evaporation rates at steady state. The consistency of the experimental data was checked using standard data reconciliation procedures, under the constraint that elemental conservation relations were satisfied [31,32]. No proof of mismatch was found for calculated rates. Under pure aerobic methylotrophic growth, the only products produced by B. methanolicus were biomass and $\mathrm{CO}_{2}$ (Table 3). In these conditions, methanol was almost completely consumed by the cells and less than $3 \%$ was evaporated. As expected, oxygen consumption was higher than $\mathrm{CO}_{2}$ production, leading to a RQ coefficient lower than 1 , which is characteristic of reduced carbon sources like methanol [3]. Interestingly, biomass yield increased with an increase in the growth rate by reducing the production of $\mathrm{CO}_{2}$. In general, the resulting physiological parameters were in agreement with previously published data obtained in the same conditions (Table 3) [38].
Table 3 Physiological parameters of B. methanolicus MGA3 chemostat cultures growing at two different dilution rates

\begin{tabular}{|c|c|c|c|}
\hline & $\begin{array}{l}\mathrm{D}: 010 \mathrm{~h}^{-1 \mathrm{a}} \\
\text { Value } \pm \mathrm{sd}\end{array}$ & $\begin{array}{l}\text { D: } 015 h^{-1 a} \\
\text { Value } \pm s d\end{array}$ & From $[38]^{\mathrm{a}}$ \\
\hline Methanol [mmol/(gDCW*h)] & $-7.48 \pm 0.13$ & $-8.59 \pm 0.17$ & -15.5 \\
\hline Biomass $[1 / \mathrm{h}]$ & $0.100 \pm 0.03$ & $0.148 \pm 0.001$ & 0.25 \\
\hline CER $\left[\mathrm{mmol} /\left(\mathrm{gDCW} \mathrm{H}^{*}\right)\right]$ & $3.22 \pm 0.2$ & $2.60 \pm 0.13$ & 4.17 \\
\hline OUR $\left[\mathrm{mmol} /\left(\mathrm{gDCW}^{*} \mathrm{~h}\right)\right]$ & $-7.05 \pm 0.65$ & $-6.76 \pm 0.23$ & -10.82 \\
\hline $\operatorname{MER}\left[\mathrm{mmol} /\left(\mathrm{gDCW} \mathrm{W}^{*} \mathrm{~h}\right)\right]$ & $0.18 \pm 0.07$ & $0.08 \pm 0.02$ & - \\
\hline $\mathrm{RQ}\left[\mathrm{mol} \mathrm{CO} / \mathrm{mol} \mathrm{O}_{2}\right]$ & $0.46 \pm 0.01$ & $0.38 \pm 0.01$ & 0.39 \\
\hline Yield [gDCW/gMeOH] & $0.42 \pm 0.02$ & $0.53 \pm 0.02$ & 0.5 \\
\hline
\end{tabular}

sd standard deviation; $C E R \mathrm{CO}_{2}$ evolution rate; $\mathrm{OUR} \mathrm{O}_{2}$ uptake rate; $M E R$ methanol evaporation rate; $R Q$ respiratory coefficient

a Positive values mean production and negative values mean consumption

\section{Intracellular metabolome}

Comparisons of the intracellular metabolite pools of $B$. methanolicus grown at different growth rates showed no significant differences, except in the case of citric acid (Cit) and PEP, where intracellular metabolites were higher at lower growth rates (Table 4). This is probably due to the low difference between the two tested conditions. However, similar features of methanol metabolism were observed as previously observed $[39,40]$. Fructose1,6-bisphosphate (FBP) was the most abundant metabolite 
Table 4 Levels of intracellular metabolites and adenylate energy charge (AEC) of $B$. methanolicus MGA grown on methanol at D: $0.15 h^{-1}$ and $0.10 h^{-1}$

\begin{tabular}{|c|c|c|c|c|c|}
\hline \multicolumn{6}{|c|}{ Intracellular metabolite pools $[\mu \mathrm{mol} / \mathrm{gDCW} \pm \mathrm{se}]$} \\
\hline \multirow[t]{2}{*}{ PEP } & $D: 0.10 h^{-1}$ & $D: 0.10 h^{-1}$ & $D: 0.15 h^{-1}$ & $D: 0.15 h^{-1}$ & From [39] \\
\hline & $0.85 \pm 0.05$ & $1.12 \pm 0.11$ & $0.55 \pm 0.05$ & $0.48 \pm 0.02$ & \\
\hline P-Ser & $0.18 \pm 0.01$ & $0.25 \pm 0.03$ & $0.26 \pm 0.00$ & $0.28 \pm 0.04$ & \\
\hline 23PG & $2.40 \pm 0.11$ & $2.98 \pm 0.29$ & $2.05 \pm 0.15$ & $2.21 \pm 0.08$ & \\
\hline R5P & $2.18 \pm 0.36$ & $3.59 \pm 0.38$ & $3.29 \pm 0.16$ & $2.94 \pm 0.53$ & $0.49 \pm 0.09$ \\
\hline Shi3P & $0.07 \pm 0.01$ & $0.08 \pm 0.01$ & $0.09 \pm 0.01$ & $0.10 \pm 0.01$ & \\
\hline F1P & $0.24 \pm 0.06$ & $0.37 \pm 0.05$ & $0.18 \pm 0.02$ & $0.15 \pm 0.06$ & \\
\hline G6P & $0.97 \pm 0.38$ & $1.73 \pm 0.32$ & $1.04 \pm 0.53$ & $1.38 \pm 0.52$ & $2.86 \pm 0.25^{\mathrm{a}}$ \\
\hline F6P & $1.58 \pm 0.92$ & $3.54 \pm 1.18$ & $1.33 \pm 0.48$ & $1.70 \pm 0.39$ & \\
\hline M6P & $0.22 \pm 0.04$ & $0.34 \pm 0.04$ & $0.40 \pm 0.02$ & $0.45 \pm 0.07$ & \\
\hline $6 \mathrm{PG}$ & $2.25 \pm 0.25$ & $3.80 \pm 0.63$ & $1.90 \pm 0.08$ & $1.71 \pm 0.12$ & $1.62 \pm 0.13$ \\
\hline S7P & $4.45 \pm 0.11$ & $5.62 \pm 0.70$ & $4.04 \pm 0.19$ & $3.45 \pm 0.15$ & $3.55 \pm 0.31$ \\
\hline FBP & $14.61 \pm 1.30$ & $19.35 \pm 1.21$ & $14.65 \pm 0.28$ & $13.80 \pm 1.89$ & $16.28 \pm 1.30$ \\
\hline AMP & $0.64 \pm 2.42$ & $1.75 \pm 1.34$ & $1.02 \pm 0.19$ & $1.35 \pm 0.46$ & \\
\hline ADP & $5.47 \pm 0.53$ & $6.31 \pm 0.58$ & $5.04 \pm 0.17$ & $5.44 \pm 0.57$ & \\
\hline ATP & $6.79 \pm 0.13$ & $7.33 \pm 0.32$ & $7.33 \pm 0.39$ & $6.33 \pm 0.45$ & \\
\hline Fum & $0.02 \pm 0.51$ & $0.04 \pm 0.30$ & $0.20 \pm 0.09$ & $0.89 \pm 0.21$ & \\
\hline Mal & $0.41 \pm 0.35$ & $0.48 \pm 0.42$ & $0.47 \pm 0.09$ & $0.65 \pm 0.02$ & \\
\hline Cit & $4.10 \pm 0.39$ & $3.70 \pm 0.52$ & $2.87 \pm 0.08$ & $2.31 \pm 0.35$ & \\
\hline AEC & $0.74 \pm 0.26$ & $0.68 \pm 0.15$ & $0.74 \pm 0.07$ & $0.69 \pm 0.13$ & \\
\hline
\end{tabular}

Each chemostat culture was considered separately. The metabolite abbreviations and the raw data are given in Additional file 1

a This is the concentration of the pool of hexose-6-phosphate

irrespective of the culture conditions and in the same order of magnitude as in a previous metabolomics dataset obtained for this specific methylotroph grown in batch on methanol at $50{ }^{\circ} \mathrm{C}$ (Table 4, [39]). These data are consistent with the low affinity for FBP of plasmidic fructose 1,6-bisphosphatase isoenzymes upregulated upon methanol growth $[6,8,40]$. Despite the low precision of hexose6-phosphate (H6P) values due to the significant quantities outside the cell (Table 2), their sum (G6P + F6P + M6P) was quiet close to amount of H6P observed in the previous dataset. Except for pentose-5-phosphate pool (R5P), pool sizes of the intermediates of the pentose phosphate (PP) pathway were in the same range but slightly higher than the ones observed previously [39]. This is consistent with the fact that former data were obtained by fast filtration, thus lower levels due to leakage may be expected. However, this difference may be also due to the difference in culture conditions rather than the method itself. The previous study used batch culture while the presented data were obtained in a methanol limited condition. TCA cycle metabolites were found in smaller amounts in $B$. methanolicus than in pure glycolytic metabolism, in agreement with low TCA activity in methylotrophic bacteria [41-43]. Finally, the calculated adenylate energy charges (AECs) were slightly below but not significantly different from 0.80 , a value considered as characteristic of energetically healthy cells [44]. Overall our procedure resulted in reliable and reproducible data that are not significantly different from those published previously, supporting the validity of our protocol.

\section{Conclusion}

We demonstrated that, like in many prokaryotic cells, significant leakage of metabolites occurs in B. methanolicus, thus might hampering the use of protocols which include cell separation for metabolomics. For proper quantitative metabolomics studies in this methylotrophic organism, total broth quenching with correction for the metabolites present in the extracellular medium proved to be a good alternative and might be an improvement on fast filtration based approach. The use of this protocol for steady-state chemostat cultures yielded accurate, reliable and valuable datasets to assess the use of methanol by B. methanolicus at $50{ }^{\circ} \mathrm{C}$. These results are the first step toward the better system-level understanding of methanol-derived metabolism in this thermophilic gram-positive bacterium. We focused in this study metabolites closely related with the central methanol metabolism; however more quenching and extracting methods must be tested to expand the number of metabolites that could be measured. Anyway, 
by providing access to the metabolome of $B$. methanolicus in a quantitative manner, this work paves the way to rationally shape its metabolism for the efficient use of methanol as raw material in biotechnology.

\section{Additional file}

Additional file 1: List of metabolites with their abbreviations and Inchl Code and Inchl Key, raw data (i.e. analyte peak area and the corresponding concentration) and calibration coefficients.

\section{Abbreviations}

S7P: sedoheptulose 7-phosphate; PEP: phosphoenolpyruvate; 23 PG: $2+3$ phosphoglycerate; R5P: ribose 5-phosphate + ribulose 5-phosphate + xylulose 5-phosphate; G6P: glucose 6-phosphate; F1P: fructose 1-phosphate; F6P: fructose 6-phosphate; M6P: mannose 6-phosphate; 6PG: 6-phosphogluconate; FBP: fructose-16-bisphosphate; AMP: adenosine $5^{\prime}$-monophosphate; ADP: adenosine diphosphate; ATP: adenosine triphosphate; P-serine: O-L-phosphoserine; Shi3P: shikimate 3P; Fum: fumarate; Mal: malate; Cit: citric acid; RuMP: ribulose monophosphate.

\section{Authors' contributions}

MC designed, conceived the study and performed experimental studies and data analysis. GV participated in the experimental studies and data analysis. TB and JCP contributed to the data analysis. SH participated in the design, contributed to the data analysis and coordinated the project. All the authors contributed to manuscript writing. All the authors have read and approved the manuscript for publication.

\section{Author details}

1 Université de Toulouse; INSA, UPS, INP; LISBP, 135 Avenue de Rangueil, 31077 Toulouse, France. ${ }^{2}$ INRA, UMR792, Ingénierie des Systèmes Biologiques et des Procédés, 31400 Toulouse, France. ${ }^{3}$ CNRS, UMR5504, 31400 Toulouse, France. ${ }^{4}$ Department of Biotechnology, Norwegian University of Science and Technology, Trondheim, Norway. ${ }^{5}$ LISBP-INSA de Toulouse, 135 Avenue de Rangueil, 31077 Toulouse Cedex 04, France.

\section{Acknowledgements}

The authors are grateful to Serguei Sokol and Fabien Letisse for critical discussions and suggestions. MetaToul (Metabolomics \& Fluxomics Facitilies, Toulouse, France, http://www.metatoul.fr) and its staff members Edern Cahoreau, Lara Gales, Lindsay Peyriga and Hanna Barbier are gratefully acknowledged for technical support and access to mass spectrometry facilities. MetaToul is part of the national infrastructure MetaboHUB-ANR-11-INBS-0010 (The French National infrastructure for metabolomics and fluxomics, http://www.metabohub.fr). MetaToul is supported by grants from the Région Midi-Pyrénées, the European Regional Development Fund, SICOVAL, the Infrastructures en Biologie Sante et Agronomie (IBiSa, France), the Centre National de la Recherche Scientifique (CNRS) and the Institut National de la Recherche Agronomique (INRA). This work was funded by PROMYSE European project (FP7-KBBE-2011-3-6-04).

\section{Competing interests}

The authors declare that they have no competing interests.

\section{Human and animal rights and informed consent}

This article does not contain any studies with human participants or animals performed by any of the authors Marc Carnicer, Gilles Vieira, Trygve Brautaset, Jean-Charles Portais and Stephanie Heux.

\section{Funding}

This work was supported by the EU-FP7 PROMYSE KBBE.2011.3.6-04. Synthetic Biology principles towards the cell factory notion in biotechnology.

Received: 14 March 2016 Accepted: 11 May 2016

Published online: 01 June 2016

\section{References}

1. Schrader J, Schilling M, Holtmann D, Sell D, Filho MV, Marx A, Vorholt Ja. Methanol-based industrial biotechnology: current status and future perspectives of methylotrophic bacteria. Trends Biotechnol. 2009;27:107-15.

2. Brautaset $T$, Jakobsen $\varnothing \mathrm{M}$, Josefsen KD, Flickinger MC, Ellingsen TE. Bacillus methanolicus: a candidate for industrial production of amino acids from methanol at $50^{\circ} \mathrm{C}$. Appl Microbiol Biotechnol. 2007;74:22-34.

3. Müller JEN, Heggeset TMB, Wendisch VF, Vorholt Ja, Brautaset T. Methylotrophy in the thermophilic Bacillus methanolicus, basic insights and application for commodity production from methanol. Appl Microbiol Biotechnol. 2014;99:535-51.

4. Naerdal I, Pfeifenschneider J, Brautaset T, Wendisch VF. Methanol-based cadaverine production by genetically engineered Bacillus methanolicus strains. Microb Biotechnol. 2015;8:342-50.

5. Anthony C. The biochemistry of methylotrophs. London: Academic Press; 1982.

6. Heggeset TMB, Krog A, Balzer S, Wentzel A, Ellingsen TE, Brautaset T. Genome sequence of thermotolerant Bacillus methanolicus: features and regulation related to methylotrophy and production of L-lysine and L-glutamate from methanol. Appl Environ Microbiol. 2012;78:5170-81.

7. Markert B, Stolzenberger J, Brautaset T, Wendisch VF. Characterization of two transketolases encoded on the chromosome and the plasmid pBM19 of the facultative ribulose monophosphate cycle methylotroph Bacillus methanolicus. BMC Microbiol. 2014;14:7-7.

8. Stolzenberger J, Lindner SN, Persicke M, Brautaset T, Wendisch VF. Characterization of fructose 1,6-bisphosphatase and sedoheptulose 1,7-bisphosphatase from the facultative ribulose monophosphate cycle methylotroph Bacillus methanolicus. J Bacteriol. 2013;195:5112-22.

9. Stolzenberger J, Lindner SN, Wendisch VF. The methylotrophic Bacillus methanolicus MGA3 possesses two distinct fructose 1,6-bisphosphate aldolases. Microbiol. 2013;159:1770-81.

10. Irla M, Neshat A, Brautaset T, Rückert C, Kalinowski J, Wendisch VF. Transcriptome analysis of thermophilic methylotrophic Bacillus methanolicus MGA3 using RNA-sequencing provides detailed insights into its previously uncharted transcriptional landscape. BMC Genom. 2015;16:1-22.

11. Müller JEN, Litsanov B, Bortfeld-Miller M, Trachsel C, Grossmann J, Brautaset T, Vorholt JA. Proteomic analysis of the thermophilic methylotroph Bacillus methanolicus MGA3. Proteomics. 2014;14:725-37.

12. Heux S, Meynial-Salles I, O'Donohue MJ, Dumon C. White biotechnology: state of the art strategies for the development of biocatalysts for biorefining. Biotechnol Adv. 2015;33:1653-70.

13. van Der Werf MJ, Jellema RH, Hankemeier T. Microbial metabolomics: replacing trial-and-error by the unbiased selection and ranking of targets. J Ind Microbiol Biotechnol. 2005;32:234-52.

14. van Gulik WM. Fast sampling for quantitative microbial metabolomics. Curr Opin Biotechnol. 2010;21:27-34.

15. Taymaz-Nikerel H, de Mey M, Ras C, ten Pierick A, Seifar RM, van Dam JC, Heijnen JJ, van GulikWM. Development and application of a differential method for reliable metabolome analysis in Escherichia coli. Anal Biochem. 2009;386:9-19.

16. Wittmann C, Krömer JO, Kiefer P, Binz T, Heinzle E. Impact of the cold shock phenomenon on quantification of intracellular metabolites in bacteria. Anal Biochem. 2004;327:135-9.

17. Canelas AB, Ras C, ten Pierick A, van Dam JC, Heijnen JJ, van GulikWM. Leakage-free rapid quenching technique for yeast metabolomics. Metabolomics. 2008:4:226-39.

18. Carnicer M, Canelas AB, Pierick A, Zeng Z, Dam J, Albiol J, Ferrer P, Heijnen JJ, Gulik W. Development of quantitative metabolomics for Pichia pastoris. Metabolomics. 2012;8:284-98

19. Villas-Bôas SG, Bruheim P. Cold glycerol-saline: the promising quenching solution for accurate intracellular metabolite analysis of microbial cells. Anal Biochem. 2007;370:87-97.

20. de Jonge LP, Douma RD, Heijnen JJ, van GulikWM. Optimization of cold methanol quenching for quantitative metabolomics of Penicillium chrysogenum. Metabolomics. 2012;8:727-35.

21. Bolten CJ, Kiefer P, Letisse F, Portais J-C, Wittmann C. Sampling for metabolome analysis of microorganisms. Anal Chem. 2007;79:3843-9. 
22. Millard P, Massou S, Wittmann C, Portais J-C, Létisse F. Sampling of intracellular metabolites for stationary and non-stationary ${ }^{13} \mathrm{C}$-metabolic flux analysis in Escherichia coli. Anal Biochem. 2014;465:38-49.

23. Schaub J, Schiesling C, Reuss M, Dauner M. Integrated sampling procedure for metabolome analysis. Biotechnol Prog. 2006;22:1434-42.

24. Rabinowitz JD, Kimball E. Acidic acetonitrile for cellular metabolome extraction from Escherichia coli. Anal Chem. 2007;79:6167-73.

25. Mashego MR, van Gulik WM, Vinke JL, Heijnen JJ. Critical evaluation of sampling techniques for residual glucose determination in carbon-limited chemostat culture of Saccharomyces cerevisiae. Biotechnol Bioeng. 2003:83:395-9.

26. Theobald U, Mailinger W, Baltes M, Rizzi M, Reuss M. In vivo analysis of metabolic dynamics in Saccharomyces cerevisiae : I. Experimental observations. Biotechnol Bioeng. 1997;55:305-16.

27. Brautaset T, Williams MD, Dillingham RD, Kaufmann C, Bennaars A, Crabbe E, Flickinger MC. Role of the Bacillus methanolicus citrate synthase II gene, citY, in regulating the secretion of glutamate in L-lysine-secreting mutants. Appl Environ Microbiol. 2003;69:3986-95.

28. Kiefer P, Nicolas C, Letisse F, Portais J-C. Determination of carbon labeling distribution of intracellular metabolites from single fragment ions by ion chromatography tandem mass spectrometry. Anal Biochem. 2007;360:182-8

29. Mashego, Wu L, van Dam JC, Ras C, Vinke JL, van Winden Wa, van Gulik WM, Heijnen JJ. MIRACLE: mass isotopomer ratio analysis of U-13C-labeled extracts. A new method for accurate quantification of changes in concentrations of intracellular metabolites. Biotechnol Bioeng. 2004;85:620-8.

30. R Development Core Team: $\mathrm{R}$ : a language and environment for statistical computing. pp. http://www.R-project.org: R Foundation for Statistical Computing, Vienna, Austria; 2013: http://www.R-project.org.

31. van der Heijden RT, Heijnen JJ, Hellinga C, Romein B, Luyben KC. Linear constraint relations in biochemical reaction systems: I. Classification of the calculability and the balanceability of conversion rates. Biotechnol Bioeng. 1994;43:3-10.

32. Verheijen PJT: Data Reconciliation and Error Detection. Edited by Smolke CD2010: 8.1-8.13.

33. Dauner M, Storni T, Sauer U. Bacillus subtilis metabolism and energetics in carbon-limited and excess-carbon chemostat culture. J Bacteriol. 2001;183:7308-317.
34. Stephanopoulos GN, Aristidou AA. Material balances and data consistency. Nielsen J: Academic Press; 1997. p. 115-46.

35. Douma RD, de Jonge LP, Jonker CTH, Seifar RM, Heijnen JJ, van Gulik WM. Intracellular metabolite determination in the presence of extracellular abundance: application to the penicillin biosynthesis pathway in Penicillium chrysogenum. Biotechnol Bioeng. 2010;107:105-15.

36. Wellerdiek M, Winterhoff D, Reule W, Brandner J, Oldiges M. Metabolic quenching of Corynebacterium glutamicum: efficiency of methods and impact of cold shock. Bioprocess Biosyst Eng. 2009;32:581-92.

37. Paczia N, Nilgen A, Lehmann T, Gatgens J, Wiechert W, Noack S. Extensive exometabolome analysis reveals extended overflow metabolism in various microorganisms. Microb Cell Fact. 2012;11:122.

38. Pluschkell SB, Flickinger MC. Dissimilation of ${ }^{13} \mathrm{C}$ methanol by continuous cultures of Bacillus methanolicus MGA3 at $50{ }^{\circ} \mathrm{C}$ studied by ${ }^{13} \mathrm{C}$ NMR and isotope-ratio mass spectrometry. Microbiology. 2002;148:3223-33.

39. Müller JE, Meyer F, Litsanov B, Kiefer P, Vorholt JA. Core pathways operating during methylotrophy of Bacillus methanolicus MGA3 and induction of a bacillithiol-dependent detoxification pathway upon formaldehyde stress. Mol Microbiol. 2015. doi:10.1111/mmi.13200-13210.11111/ mmi.13200.

40. Bozdag A, Komives C, Flickinger MC. Growth of Bacillus methanolicus in $2 \mathrm{M}$ methanol at $50^{\circ} \mathrm{C}$ : the effect of high methanol concentration on gene regulation of enzymes involved in formaldehyde detoxification by the ribulose monophosphate pathway. J Ind Microbiol Biotechnol. 2015:42:1027-38.

41. Chistoserdova L, Kalyuzhnaya MG, Lidstrom ME. The expanding world of methylotrophic metabolism. Annu Rev Microbiol. 2010;63:477-99.

42. Peyraud R, Kiefer P, Christen P, Massou S, Portais J-C, Vorholt Ja. Demonstration of the ethylmalonyl-CoA pathway by using ${ }^{13} \mathrm{C}$ metabolomics. Proc Natl Acad Sci USA. 2009;106:4846-51.

43. Peyraud R, Schneider K, Kiefer P, Massou S, Vorholt Ja, Portais J-C. Genome-scale reconstruction and system level investigation of the metabolic network of Methylobacterium extorquens AM1. BMC Syst Biol. 2011;5:189-189.

44. Chapman AG, Fall L, Atkinson DE. Adenylate energy charge in Escherichia coli during growth and starvation. J Bacteriol. 1971;108:1072-86.

\section{Submit your next manuscript to BioMed Central and we will help you at every step:}

- We accept pre-submission inquiries

- Our selector tool helps you to find the most relevant journal

- We provide round the clock customer support

- Convenient online submission

- Thorough peer review

- Inclusion in PubMed and all major indexing services

- Maximum visibility for your research

Submit your manuscript at www.biomedcentral.com/submit
() Biomed Central 